\title{
Computerized Clinical Pharmacy Management System Improves Prescribing Quality and Rational Antimicribial Use
}

\author{
Xianhua Ren, Quansheng Ren* \\ Department of Pharmacy, Affiliated Hospital of Inner Mongolia Medical University, Hohhot, People's Republic of China
}

Email address:

renxianhua237@163.com (Xianhua Ren)

${ }^{*}$ Corresponding author

To cite this article:

Xianhua Ren, Quansheng Ren. Computerized Clinical Pharmacy Management System Improves Prescribing Quality and Rational Antimicribial Use. American Journal of Internal Medicine. Vol. 4, No. 6, 2016, pp. 107-112. doi: 10.11648/j.ajim.20160406.13

Received: October 27, 2016; Accepted: November 22, 2016; Published: November 24, 2016

\begin{abstract}
To introduce the increased efficiency and improved health care provision brought by incorporating a certified clinical pharmacy management (CPM) system into an existing hospital information system. Evaluate the appropriateness rate of prescriptions or orders, the rational use of antimicrobial use, and the workflow of clinical pharmacist before and after the adoption of the CPM system. The adoption of the CPM system markedly increased the appropriateness rate of prescriptions and orders by $89 \%$ in 2011 as compared to that of 2010 . The CPMS decrease the antimicrobial use both in outpatients and inpatients by $47.0 \%$ and 51.9 respectively in December 2011 as compared to that of January 2011. The CPMS also streamlined the clinical pharmacist's workflow and help disseminate medication use knowledge and policies within the hospital.
\end{abstract}

Keywords: Clinical Pharmacy Management System, Prescribing Appropriateness Rate, Rational Antimicrobial Use

\section{Introduction}

With the ever-evolving development in computer software and networking technologies, many hospitals in China have gone through a revolution in processing healthcare-related information. Likewise, this is the case in our hospital, which is a Grade 3A hospital (the top grade according to China's hospital grading system) located in the capital of Inner Mongolia, China. The hospital possesses 34 clinical departments, a ward equipped with more than 1800 beds, and an on-site pharmacy. The hospital serves populations of various ethnicities with yearly hospital admissions of patients exceeding one million. Aiming to tackle the constant pressure of providing efficient and high quality healthcare services, our hospital deployed computerized hospital information (HI) system in 2005. We avoid using the term Computer Physician Order Entry (CPOE) to describe the original HI system, for it did not conform to the commonly-accepted definition of CPOE. Actually, the HI system had two modules with one module for maintaining patient medical records and another module for e-prescribing. Before 2011, the existing HI system did not have a functioning pharmaceutical subsystem, for laboratory tests, radiology images, and other various information relating to healthcare provision were not incorporated into the HI system, resulting in inconvenience and inefficiency in the overall healthcare provision.

Our hospital maintains a staff of seventy members in the Department of Pharmacy, among whom five members are clinical pharmacists. Our department has underwent the reform process in the provision of pharmaceutical practice and paradigm shift of moving away from compounder and supplier of medicinal products towards a more inclusive focus on patient care, as advocated by WHO [1]. In recent years, the healthcare agencies of Chinese government has played proactive roles in regulating healthcare provision, exemplified by the mandate of the Regulations on pharmacy administration in medical organizations [2, 3], in which pharmacists were required to assume more responsibilities including formulary management, setting up standard treatment guidelines, and various aspects relating to decreasing adverse drug events and facilitating rational medication use.

While applauding the critical changes occurred in the pharmacy practice, we found it challenging to undertake the 
responsibilities partly due to lack of technical means. Before 2011, the existing HI system had major shortcomings, failing to provide the needed help on many different levels. Firstly, the Drug and Therapeutics Committee (DTC) of our hospital maintained a list of essential medicines based on the National Essential Medicine List (NEML) [4], but the list existed as a separate document file, not being incorporated into the existing HI system. Therefore, it was inconvenient and inefficient for physicians and pharmacists to consult when needed. Secondly, though regulatory authorities issued Chinese National Formulary [5] for physicians and pharmacists as standard treatment guidelines (STGs), the STGs were not incorporated into the existing HI system, resulting in inconvenience and poor compliance. Thirdly, drug-drug, and drug-allergy interactions were not checked at the time of prescribing due to lack of real-time decision support system. Therefore, the evaluation of all prescriptions (referred to as "pharmacy validation" thereafter) rested with pharmacists at the time of dispensing, which involved pharmacists in a huge workload to evaluate prescriptions without convenient reference to computerized NEML [4] and STGs [5]. Finally but not lastly, pharmacists have been required to take on more responsibilities regarding pharmaceutical public health. For example, regulatory authorities issued the Regulations on prescribing evaluation in hospitals [6], targeting medication errors occurred nationwide, which involved pharmacists in more workload, for it often took hours for a pharmacist to search and extract the needed medical information for an individual case before reporting the monthly evaluation results to regulatory agencies.

As antimicrobial resistance (AMR) has been elevated to national focus, Chinese government plays significant roles in addressing irrational antimicrobial misuse including policy making, strategy implementing, and outcome monitoring, just as WHO advocated "The emergence of AMR is a complex problem driven by many inter-connected factors; single, isolated interventions have little impact. A global and national multi-sectoral response is urgently needed to combat the growing threat of AMR." Antimicrobial treatments are further classified as therapeutic antibiotic and prophylactic antibiotic treatments. Therapeutic antibiotic treatment refers to the use of substances that reduce the growth or reproduction of bacteria, including eradication therapy. Prophylactic antibiotic treatment refers to the use of antibiotics before, during, or after diagnostic, therapeutic, or surgical procedure to prevent infectious complications. In recent years, Chinese government has exerted a series of regulations aiming to contain AMR nationwide. The Principles of clinical antimicrobial usage [7] was issued in 2004, serving as guidelines for antimicrobial usage for therapeutic antimicrobial treatment purpose in major specialties. The Principles of clinical antimicrobial usage [8] was issued in 2009, providing guidelines for prophylactic antibiotic treatment involved in major surgical procedures. In the same year, Principles of perioperative prophylactic treatment in clean surgeries [9] was issued, outlining the principles of antimicrobial usage specific to clean surgical procedures. These guidelines required mandatory participation and mandated that pharmacists be responsible for the outcome of antimicrobial usage, which involved pharmacists in challenging responsibilities, especially before 2011.

Obviously, changes were required. The leadership of our hospital set out to employ computer and network technologies to normalize the provision and management of pharmaceutical practice at the beginning of 2010. Through extensive investigation and evaluation, a modified version of clinical pharmacy management (CPM) system was selected, which was developed by a certified medical information management company (Medicom Company, Chengdu, China) and went live on a full scale on January 1, 2011. The CPM system was designed as a middleware, being seamlessly incorporated into the existing HI system with significant advantages that help clinical physicians and pharmacists manage and streamline their workflow. Firstly, NEML [10] and the STGs [11] were intelligently incorporated into the CPM system. Therefore, physicians can acquire the needed information without effort to consult a separate document file in PDF format with more than 1000 pages under the intense face-to-face observation of involved patients. Secondly, real-time pharmacy validation of prescribing becomes a reality, for the CPM system monitors every prescribing generated to check the drug-drug and drug-allergy interactions with reference to the medical record of individual patients, thus ensuring that medicines of appropriate indication and assured safety are delivered. Thirdly, the regulations and principles regulating antimicrobial usage $[10,11]$ are incorporated into the CPM system as guidelines for antimicrobial prescribing, making real-time pharmacy validation on antimicrobial usage possible. Finally, the CPM system streamlines the workflow for clinical pharmacists to prepare and report the results of prescribing evaluation and antimicrobial usage evaluation to regulatory agencies by providing customized query-building options depending on specific sampling method.

In the following, the advantages brought by the CPM system will be exemplified by a cohort study relating to the prescribing appropriateness rate and antimicrobial usage by analyzing data of the year 2010 and 2011.

\section{Methods}

\subsection{Settings and Subjects}

This study was conducted both in outpatient and in inpatient settings within the hospital by analyzing medical records from the year 2010 and 2011. Indicators of prescribing appropriateness rate and antimicrobial usage were employed to evaluate the impacts of the CPM system. The medical records of 2010 were the same as the monthly prescription evaluation reports that were submitted to regulatory authorities. The medical records of 2011 included the whole of 418446 prescriptions generated within the hospital, in which 335857 prescriptions were from outpatient settings and 82,589 orders were from inpatient settings. The average age of the subjects of 2011 was $46 \pm 16$ years old. Among the total subjects, 194875 
subjects were male and 223571 subjects were female.

\subsection{Assessing Prescribing Appropriateness Rate}

Criteria for judging whether prescribing was appropriate were the same in 2010 and 2011 as defined by the Regulations [5], in which all types of prescribing errors are divided into three classes: (1) non-standard prescription, which includes 15 sub-classes with numbers assigned from 1.11 to 1.15 ; (2) inappropriate medication, which includes 9 sub-classes with numbers assigned from 2.1 to 2.9 ; (3) abnormal prescription, which contains 4 sub-classes with numbers assigned from 3.1 to 3.4 (Table 1). The assigned number is used as shorthand for corresponding prescribing error when we prepare and report the prescribing evaluation results to regulatory agencies.

Table 1. Assessing prescribing appropriateness rate.

\begin{tabular}{|c|c|c|c|c|c|c|c|c|c|c|c|c|}
\hline & January & & February & & March & & April & & May & & June & \\
\hline $\begin{array}{l}\text { Prescription } \\
\text { source }\end{array}$ & Outpatient & Inpatient & Outpatient & Inpatient & Outpatient & Inpatient & Outpatient & Inpatient & Outpatient & Inpatient & Outpatient & Inpatient \\
\hline $\begin{array}{l}\text { Qualified } \\
\text { prescription }\end{array}$ & 11898 & 4468 & 11678 & 3482 & 16904 & 4683 & 18779 & 5199 & 20311 & 5735 & 19268 & 5342 \\
\hline $\begin{array}{l}\text { Total } \\
\text { prescription }\end{array}$ & 24532 & 6842 & 20596 & 5024 & 28699 & 6877 & 30047 & 7016 & 30635 & 7468 & 27487 & 7104 \\
\hline $\begin{array}{l}\text { Qualified rate } \\
(\%)\end{array}$ & 48.5 & 65.3 & 56.7 & 69.3 & 58.9 & 68.1 & 62.5 & 74.1 & 66.3 & 76.8 & 70.1 & 75.2 \\
\hline
\end{tabular}

Table 1. Continue.

\begin{tabular}{|c|c|c|c|c|c|c|c|c|c|c|c|c|}
\hline & July & & August & & September & & October & & November & & December & \\
\hline $\begin{array}{l}\text { Prescription } \\
\text { source }\end{array}$ & Outpatient & Inpatient & Outpatient & Inpatient & Outpatient & Inpatient & Outpatient & Inpatient & Outpatient & Inpatient & Outpatient & Inpatient \\
\hline $\begin{array}{l}\text { Qualified } \\
\text { prescription }\end{array}$ & 21893 & 5565 & 24244 & 5962 & 26503 & 5995 & 27287 & 6287 & 25859 & 6158 & 27713 & 6439 \\
\hline $\begin{array}{l}\text { Total } \\
\text { prescription }\end{array}$ & 26473 & 6947 & 28489 & 7039 & 29981 & 7146 & 30185 & 7177 & 28479 & 6958 & 30254 & 6991 \\
\hline $\begin{array}{l}\text { Qualified rate } \\
(\%)\end{array}$ & 82.7 & 80.1 & 85.1 & 84.7 & 88.4 & 83.9 & 90.4 & 87.6 & 90.8 & 88.5 & 91.6 & 92.1 \\
\hline
\end{tabular}

This assessment was conducted in two steps. First, prescribing appropriateness rates of 2010 and 2011 were compared using the same sampling method as defined by the Regulations, in which a total of 130 prescribing evaluations on 100 random prescribing samples from outpatients and 30 random prescribing samples from inpatients are required to be submitted to regulatory agencies on a monthly basis. Second, the monthly prescribing appropriateness rates of 2011 were compared both in outpatient and in inpatient settings using large sampling method.

\subsection{Assessing Prophylactic Antibiotic Treatment for Clean Surgical Procedures}

Though the Guidelines manifesting the antimicrobial selection criteria for clean surgical procedures were available to surgeons and clinical pharmacists both in 2010 and in 2011, it began to serve as a functional decision support system after the introduction of the CPM system. This assessment was conducted by comparing the percentage of patients receiving clean surgical procedures on a monthly basis in 2011 using large sampling method.

The Guidelines outlined indication for prophylaxis, choice of agent for surgical prophylaxis, timing of administration, and clinical considerations relating to specific individual patient. In light of the Guidelines, antimicrobial prophylaxes are not recommended for clean and benign surgical procedures including head and neck surgeries, breast surgeries, vascular surgeries, hernia repair, portosystemic shunt surgeries, lienectomy, percutaneous endoscopic gastrostomy, laparoscopic cholecystectomy, endoscopic retrograde cholangiopancreatography (ERCP).

\subsection{Assessing Intensity of Antimicrobial Utilization in Inpatient Setting}

The assessment was to reflect the longitudinal change of intensity of antimicrobial usage in inpatient setting on a monthly basis in 2011 as the impact of the CPM system. Starting in January 2011, three guidelines were intelligently incorporated into the CPM system, aiming to tackle antimicrobial misuse on three different levels. Firstly, Guidelines for prophylactic antimicrobial usage was installed, as described above. Secondly, Guidelines for perioperative prophylactic treatment was also incorporated, serving as guidelines for antimicrobial prophylaxis in surgeries of clean-contaminated, contaminated, and dirty surgeries. Thirdly, Principles of clinical antimicrobial usage were also included in the CMP system, serving as guidelines for therapeutic antibiotic treatment. It outlines the procedure for diagnosing and treating bacterial infections, including acute bacterial upper respiratory tract infections, acute bacterial lower respiratory tract infections, urinary tract infections (including cystitis and pyelonephritis), bacterial prostatitis, acute infectious diarrhea, bacterial meningitis and brain abscess, septicemia, infective endocarditis, intra-abdominal infections, bone and joint infections, skin and soft tissue infections, oral and maxillofacial infections, ocular infections, 
vaginal infections, cervicitis, pelvic inflammatory diseases, sexually transmitted diseases, deep mycoses, mycobacterial infections, diphtheria, pertussis, scarlatina, pestis, anthrax, tetanus, gas gangrene, Salmonella infections, brucellosis, leptospinosis, febris recurrens, and Lyme diseases.

In this assessment, 82,589 orders from inpatient setting were analyzed for the year of 2011. Data were expressed as Defined Daily Dose. The adoption of the CPM system made this assessment possible.

\subsection{Assessing Overall Antimicrobial Utilization}

To evaluate the overall antimicrobial use both in outpatient and inpatient settings, the percentage of patients receiving antimicrobial treatment were presented and analyzed on a monthly basis. The data set for this assessment included the total of 418446 prescriptions generated in 2011.

\subsection{Statistical Analysis}

The data obtained was subjected to statistical analysis using one way ANOVA.

\section{Results}

\subsection{The CPM System Improved Prescribing Appropriateness Rates}

To make a meaningful comparison of the differences between 2010 and 2011, which were attributable to the adoption of CPMS, the same sampling method was adopted to analyze the data for 2011 as compared to that of 2010, in which 100 prescribing from inpatients and 30 prescribing from outpatients were randomly selected. The results showed the prescribing appropriate rates for both inpatients and outpatients in 2010 remained relatively over months with statistical insignificance (Table 2). In contrast, the adoption of CPMS steadily increased the prescribing appropriateness rates over months in 2011. The prescribing appropriateness rates for inpatients increased by $56.4 \%$ as compared to the same month in 2010, and prescribing appropriateness rates for outpatients increased by $164.2 \%$ as compared to the same month in 2010 .

Table 2. The proportion of antibiotic treatment in 2011 (\%).

\begin{tabular}{|c|c|c|c|c|c|c|c|c|c|c|c|c|}
\hline & Jan & Feb & Mar & Apr & May & Jun & Jul & Aug & Sep & Oct & Nov & Dec \\
\hline Inpatient & 71.3 & 56.2 & 56.0 & 54.2 & 50.9 & 48.5 & 46.6 & 42.8 & 37.2 & 38.3 & 34.3 & 31.5 \\
\hline Outpatient & 32.5 & 30.5 & 28.4 & 28.1 & 26.0 & 23.4 & 21.8 & 22.4 & 20.7 & 19.6 & 19.1 & 17.2 \\
\hline
\end{tabular}

Based on the advanced functionality provided by CPMS, longitudinal comparison over month was possible in 2011. The prescribing appropriateness rate of the total subjects, which encompassed a total of 335857 prescriptions from ambulatory clinics and 82589 orders from wards, were analyzed. The prescribing appropriateness rate for outpatients reached $91.6 \%$ in December 2011, representing an increase of $89 \%$ as compared to that of January 2011 (Table 3). In parallel, the prescribing appropriateness rate for inpatients reached $92.1 \%$ in December 2011, representing an increase of $41 \%$ as compared to that of January 2011.

Table 3. The use of prophylactic antibiotics for clean operative in 2011.

\begin{tabular}{|c|c|c|c|c|c|c|c|c|c|c|c|c|}
\hline & Jan & Feb & Mar & Apr & May & Jun & Jul & Aug & Sep & Oct & Nov & Dec \\
\hline $\begin{array}{l}\text { The proportion of } \\
\text { antibiotic treatment (\%) }\end{array}$ & 87.60 & 85.20 & 78.40 & 62.10 & 54.20 & 51.70 & 52.60 & 47.20 & 51.30 & 33.10 & 30.50 & 24.50 \\
\hline
\end{tabular}

\subsection{The CPM System Reduced the Use of Prophylactic Antibiotics for Clean Surgeries in 2011}

To evaluate the impact of CPMS on antimicrobial use in inpatients receiving clean operation in 2011, 9869 records were isolated and analyzed from the total inpatients. The outcome showed that prophylactic antibiotics for clean operation decreased steadily over months in 2011, reaching $24.5 \%$ in December, a sharp decrease of $72 \%$ as compared to that of January (Table 4).

Table 4. 2011 inpatients defined daily doses of antibiotics.

\begin{tabular}{lllllllllllll}
\hline & Jan & Feb & Mar & Apr & May & Jun & Jul & Aug & Sep & Oct & Nov & Dec \\
\hline DDD & 125.40 & 101.70 & 88.90 & 82.60 & 76.40 & 70.40 & 63.10 & 47.50 & 41.80 & 38.10 & 37.40 & 37.20 \\
\hline
\end{tabular}

\subsection{The CPM System Reduced the Intensity of Antimicrobial Use for Inpatients in 2011}

To evaluate the impact of CPMS on antimicrobial use in inpatients in 2011, 82589 orders of all the selected inpatients were analyzed with longitudinal data presented over months. The results showed that average DDDs for inpatients decreased steadily over months in 2011 , reaching $37.2 \%$ in
December, meeting the requirement set by WHO (Table 5).

\subsection{CPMS Attributed to Improved Rational Antimicrobial Use in 2011}

Due to the real-time invention of CPMS on the antimicrobial use related prescribing generated anywhere within the hospital, the use of antibiotics for inpatients and outpatients were decreased markedly. The antibiotic use 
decreased from $32.5 \%$ in January to $17.2 \%$ for outpatients, representing a decrease of $47 \%$ (Table 5). In parallel, the antibiotic use decreased from $71.3 \%$ in January to $34.3 \%$ for inpatients, representing a decrease of $51.9 \%$.

Table 5. The CPMS increases.

\begin{tabular}{|c|c|c|c|c|c|c|c|c|c|c|c|c|}
\hline \multirow[b]{2}{*}{ Time } & \multicolumn{2}{|c|}{ January } & \multicolumn{2}{|c|}{ February } & \multicolumn{2}{|c|}{ March } & \multicolumn{2}{|l|}{ April } & \multicolumn{2}{|l|}{ May } & \multicolumn{2}{|l|}{ June } \\
\hline & 2010 & 2011 & 2010 & 2011 & 2010 & 2011 & 2010 & 2011 & 2010 & 2011 & 2010 & 2011 \\
\hline Qualified rate (Outpatient \%) & 60.1 & 66.8 & 65.5 & 70.1 & 58.7 & 69.2 & 59.0 & 73.1 & 54.0 & 77.4 & 55.8 & 76.4 \\
\hline Qualified rate (Inpatient \%) & 43.0 & 47.2 & 40.5 & 57.1 & 40.9 & 57.8 & 39.4 & 63.5 & 42.6 & 65.2 & 45.1 & 71.5 \\
\hline
\end{tabular}

Table 5. Continue.

\begin{tabular}{|c|c|c|c|c|c|c|c|c|c|c|c|c|}
\hline \multirow[b]{2}{*}{ Time } & \multicolumn{2}{|l|}{ July } & \multicolumn{2}{|c|}{ August } & \multicolumn{2}{|c|}{ September } & \multicolumn{2}{|c|}{ October } & \multicolumn{2}{|c|}{ November } & \multicolumn{2}{|c|}{ December } \\
\hline & 2010 & 2011 & 2010 & 2011 & 2010 & 2011 & 2010 & 2011 & 2010 & 2011 & 2010 & 2011 \\
\hline Qualified rate (Outpatient \%) & 52.0 & 81.2 & 57.3 & 85.4 & 63.5 & 84.6 & 60.5 & 88.2 & 62.7 & 87.3 & 60.1 & 94.0 \\
\hline Qualified rate (Inpatient \%) & 41.9 & 83.4 & 45.8 & 84.9 & 43.1 & 90.1 & 44.7 & 91.3 & 45.8 & 92.0 & 47.5 & 92.5 \\
\hline
\end{tabular}

\section{Discussion}

Like other professions, the pharmacist profession does not exist in vacuum and is subject to various socio-technical factors. This study was conducted with the intention of making meaningful comparisons between the data of 2010 and 2011 when the CPM system was formally introduced. Considering the dynamic nature of healthcare provision, we are not in a position to be able to separate different determinants affecting the quality and efficiency and healthcare provision, for the Regulations and Principles mentioned above require complete compliance [12].

Medication error is a complicated issue that can be attributed to various socio-technical factors. Medication errors account for a large proportion of adverse drug events and are preventable. The CPM system provides technical means to intervene medication error at the time of prescribing with reference to the medicine use specified in the stand treatment guideline, which markedly improved the prescribing appropriateness rate, as is confirmed in previous studies Pharmacy validation together with CPOE has been demonstrated to be effective in reducing medication errors, as is advocated by WHO guideline on rational use of medicines [13]. On the other hand, the appropriateness rate in 2011 was not desirable given the absolute number of medication errors occurred. We are planning to add more functionality to the CPM system to classify and analyze medication errors. Therefore, we can have a better understanding about the priority problems relating to medication error and undertake corresponding measures to address the problem.

The need for prudent use of antimicrobials cannot be overemphasized, just as is declared by WHO [14]. We investigated the antimicrobial use from the perspectives of therapeutic treatment and prophylactic treatment, respectively. The term surgical site infection is used to encompass the surgical wound and infections involving the body cavity, bones, joints, meninges and other tissues involved in the operation. In procedures that require the insertion of implants or prosthetic devices the term also encompasses infections associated with these devices. Prophylactic administration of antibiotics inhibits growth of contaminating bacteria and their adherence to prosthetic implants, thus reducing the risk of infection [14].

\section{Conclusion}

Antimicrobial uses both for therapeutic treatment and prophylactic treatment are under stringent regulations from healthcare authorities, especially in 2011. Though the guideline for antimicrobial use was issued in 2004, physicians found it inconvenient to consult it at the time of prescribing due to limitation in the original HI system, resulting in poor adherence to the guideline. The adaptation of the CPM system effectively solved the problems and improved the adherence to national guideline. The CPM system real-time monitors the use the antibiotics prescribed anywhere within the hospital, providing solid and reliable tools to improve the management of antibiotics. We have also perceived that the adaptation of the CPM system involves changes in power structure and behavioral pattern within the institution. We also have to demonstrate the credibility of cost-effectiveness in ourselves. In future, we are planning to evaluate the adherence rate to national guideline on antimicrobial use in further studies.

\section{References}

[1] The role of the pharmacist in the health care system. Preparing the future pharmacist: Curricular development. Report of a third WHO Consultative Group on the role of the pharmacist, Vancouver, Canada, 27-29 August 1997. Geneva: World Health Organization; 1997. WHO/PHARM/97/599. Available at: http://www.who.int/medicinedocs/.

[2] State Council of the People's Republic of China (2002) Regulations on pharmacy administration in medical organizations (trial, in Chinese). State Council Document 2002(24). Beijing: State Council. Available: http://www.satcm.gov.cn/web2010/zhengw. ugongkai/zhengcefagui/falvfagui/guizhang/2010-10-07/9326.h tml. Accessed 11 January 2012. 
[3] State Council of the People's Republic of China (2011) Regulations on pharmacy administration in medical organizations (in Chinese). Beijing: State Council. State Council Document 2011(11). Available: http://www.satcm.gov.cn/web2010/zhengwugon. gkai/zhengcefagui/falvfagui/guizhang/2011-04-07/13297.html. Accessed 11 January 2012.

[4] State Council of the People's Republic of China (2009) National Essential Medicine List (in Chinese). Beijing: State Council. State Council Document 2009(69). Available: http://www.gov.cn/gzdt/2009-08/18/content_1395524.htm. Accessed 15 January 2012.

[5] State Council of the People's Republic of China (2003) Chinese National Formulary (in Chinese). Beijing: State Council.

[6] State Council of the People's Republic of China (2010) Regulations on prescribing evaluation in hospitals (trial, in Chinese). Beijing: State Council. State Council Document 2010(28). Available: http://www.gov.cn/gzdt/2010-03/04/content 1547080.htm. Accessed 14 January 2012.

[7] State Council of the People's Republic of China (2004) Principles of clinical antimicrobial usage (in Chinese). Beijing: State Council. State Council Document 2004(285). Available: http://www.moh.gov.cn/publicfiles/business/cmsresources/mo hylfwjgs/cmsrsdocument/doc5285.doc. Accessed 15 January 2012.

[8] State Council of the People's Republic of China (2009 November) Guidelines for perioperative prophylactic treatment (in Chinese). Beijing: State Council. Available: http://www.moh.gov.cn/publicfiles///business/cmsresources/m ohylfwjgs/cmsrsdocument/doc6647.doc. Accessed 15 January 2012.

[9] State Council of the People's Republic of China (2009 November) Principles of perioperative prophylactic treatment in clean surgeries (in Chinese). Beijing: State Council. Available:

http://www.moh.gov.cn/publicfiles///business/cmsresources/m ohylfwjgs/cmsrsdocument/doc6647.doc. Accessed 15 January 2012.

[10] State Council of the People's Republic of China (2007) Regulations on prescribing management (in Chinese). Beijing: State Council. State Council Document 2007 (53). Available: http://www.gov.cn/flfg/2007-03/13/content_549406.htm. Accessed 14 January 2012.

[11] State Council of the People's Republic of China (2011) Regulations on clinical antimicrobial utilizaiton (trial, in Chinese). Beijing: State Council. State Council Document 2011 (75). Available: http://bmyj.chinalaw.gov.cn/lismsPro/law download/fulltext/1 312360890480.doc. Accessed 15 January 2012.

[12] Wessling, A. and G. Boethius, Measurement of drug use in a defined population. Evaluation of the defined daily dose (DDD) methodology. Eur J Clin Pharmacol, 1990. 39 (3): p. 207-10.

[13] Zhang R, Eggleston K, Rotimi V, Zeckhauser RJ. Antibiotic resistance as a global threat: evidence from China, Kuwait and the United States. Global Health 2006; 2: 6.

[14] World Health Organization (2003) Drug and therapeutics committee: a practical guide. Geneva: World Health Organization. 